\title{
Calculations of kaonic nuclei based on chiral meson-baryon cou- pled channel interaction models
}

\author{
J. Hrtánková ${ }^{1,2, \star}$, A. Cieplý ${ }^{1}$, and J. Mareš ${ }^{1}$ \\ ${ }^{1}$ Nuclear Physics Institute, 25068 Řež, Czech Republic \\ ${ }^{2}$ Czech Technical University in Prague, Faculty of Nuclear Sciences and Physical Engineering, \\ Břehová 7, 11519 Prague 1, Czech Republic
}

\begin{abstract}
We present our latest calculations of $K^{-}$-nuclear quasi-bound states using a self-consistent scheme for constructing $K^{-}$-nuclear potentials from various subthreshold chirally inspired $\bar{K} N$ scattering amplitudes. We consider in-medium versions of the scattering amplitudes taking into account Pauli blocking in the intermediate states. The resulting $K^{-}$binding energies as well as the widths exhibit the same A dependence, however, the binding energies strongly depend on the model used.
\end{abstract}

\section{Introduction}

The aim of the present study is to compare the predictions for $K^{-}$-nuclear quasi-bound states calculated using different meson-baryon coupled channel interaction models: Prague (P NLO) [1], KyotoMunich (KM NLO) [2], Murcia (M1 and M2) [3], and Bonn (B2 and B4) [4]. They capture the physics of the $\Lambda(1405)$ and reproduce low energy $K^{-} N$ observables, including the $1 s$ level shift and width in the $K^{-}$hydrogen atom from the SIDDHARTA experiment [5]. However, the corresponding scattering amplitudes differ considerably below threshold, thus in the energy region relevant for $K^{-}$-nuclear bound-state calculations as shown in Figure 1.

\section{Model}

The binding energies $B_{K^{-}}$and widths $\Gamma_{K^{-}}$of $K^{-}$-nuclear quasi-bound states are obtained by solving the Klein-Gordon equation

$$
\left[\omega_{K^{-}}^{2}+\vec{\nabla}^{2}-m_{K^{-}}^{2}-\Pi_{K^{-}}\left(\vec{p}_{K^{-}}, \omega_{K^{-}}, \rho\right)\right] \phi_{K^{-}}=0,
$$

where $\omega_{K^{-}}=m_{K^{-}}-B_{K^{-}}-\mathrm{i} \Gamma_{K^{-}} / 2-V_{C}=\tilde{\omega}_{K^{-}}-V_{C}, m_{K^{-}}$is the $K^{-}$mass, $V_{C}$ is the Coulomb potential, and $\vec{p}_{K^{-}}$represents the kaon momentum. The self-energy operator $\Pi_{K^{-}}$is constructed in a $t \rho$ form:

$$
\Pi_{K^{-}}=2 \operatorname{Re}\left(\tilde{\omega}_{K^{-}}\right) V_{K^{-}}=-4 \pi \frac{\sqrt{s}}{m_{N}}\left(F_{0} \frac{1}{2} \rho_{p}+F_{1}\left(\frac{1}{2} \rho_{p}+\rho_{n}\right)\right),
$$

where $F_{0}$ and $F_{1}$ denote the isospin 1 and $0 \mathrm{~S}$-wave in-medium amplitudes, respectively, $m_{N}$ is the

^e-mail: hrtankova@ujf.cas.cz 

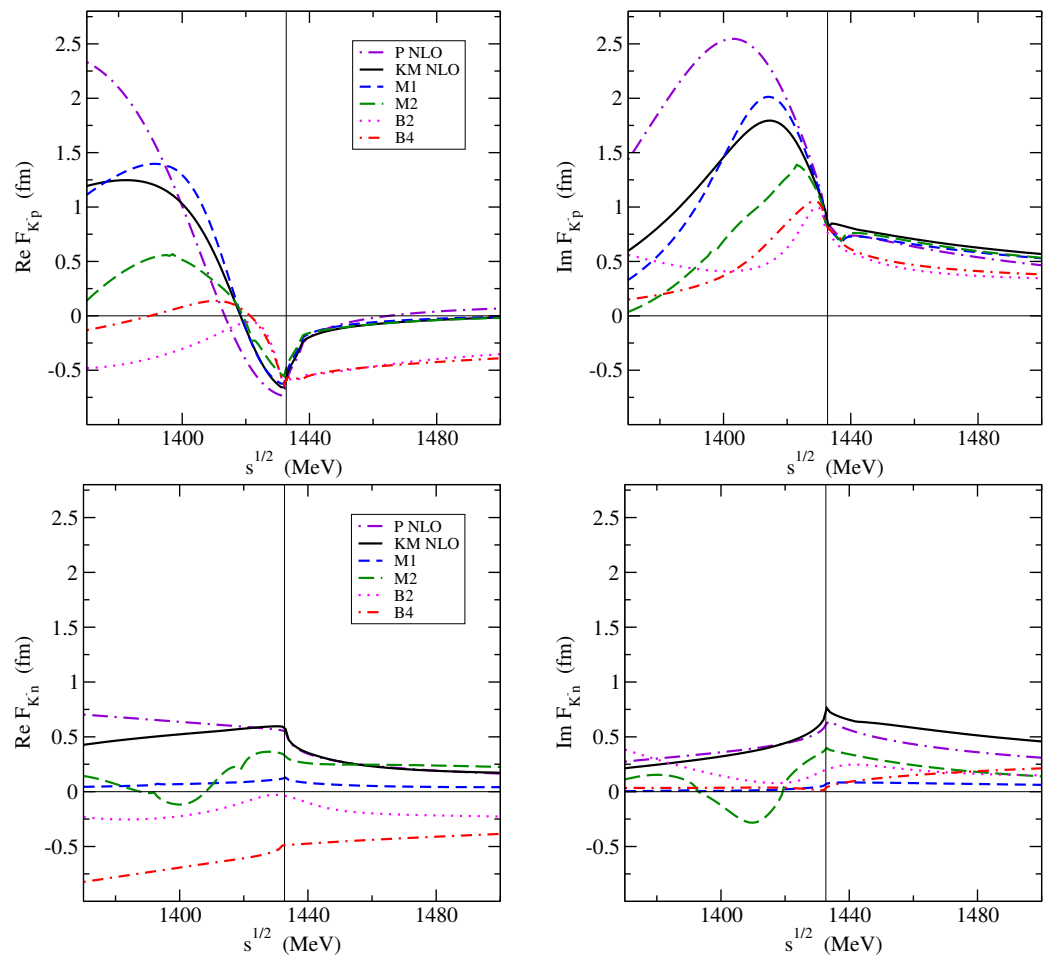

Figure 1. Energy dependence of real (left) and imaginary (right) parts of free-space $K^{-} p$ (top) and $K^{-} n$ (bottom) amplitudes in considered models.

nucleon mass and $\sqrt{s}$ is the Mandelstam variable. The proton and neutron density distributions $\rho_{p}$ and $\rho_{n}$ are obtained within a relativistic mean-field model. The in-medium amplitudes $F_{0}$ and $F_{1}$ are obtained from the free-space amplitudes by applying the multiple scattering approach (WRW) [6] which accounts for Pauli correlations:

$$
F_{1}=\frac{F_{K^{-} n}(\sqrt{s})}{1+\frac{1}{4} \xi_{k} \frac{\sqrt{s}}{m_{N}} F_{K^{-} n}(\sqrt{s}) \rho}, \quad F_{0}=\frac{\left[2 F_{K^{-}}(\sqrt{s})-F_{K^{-} n}(\sqrt{s})\right]}{1+\frac{1}{4} \xi_{k} \frac{\sqrt{s}}{m_{N}}\left[2 F_{K^{-} p}(\sqrt{s})-F_{K^{-} n}(\sqrt{s})\right] \rho},
$$

where $\xi_{k}$ is adopted from Ref. [6]. In the P NLO model [1], the integration in the underlying Green's function is limited to a certain domain due to the Pauli principle (Pauli) and the in-medium hadron self-energies (Pauli+SE) are considered as well. Figure 2 illustrates that WRW and Pauli approaches yield similar in-medium amplitudes in the subthreshold energy region relevant to our calculations.

The available energy $\sqrt{s}$ in the laboratory frame acquires the form [7] (taking into account nonnegligible contribution from particle momenta)

$$
\sqrt{s}=m_{N}+m_{K^{-}}-B_{N}-\xi_{N} B_{K^{-}}+\xi_{K^{-}} \operatorname{Re} \mathcal{V}_{K^{-}}(r)-\xi_{N} T_{N}\left(\frac{\rho}{\rho_{0}}\right)^{2 / 3},
$$

where $B_{N}$ is the average binding energy per nucleon, $\xi_{N\left(K^{-}\right)}=m_{N\left(K^{-}\right)} /\left(m_{N}+m_{K^{-}}\right), T_{N}$ is the nucleon kinetic energy determined from the Fermi Gas model, and $\mathcal{V}_{K^{-}}=V_{K^{-}}+V_{\mathrm{C}}$. 

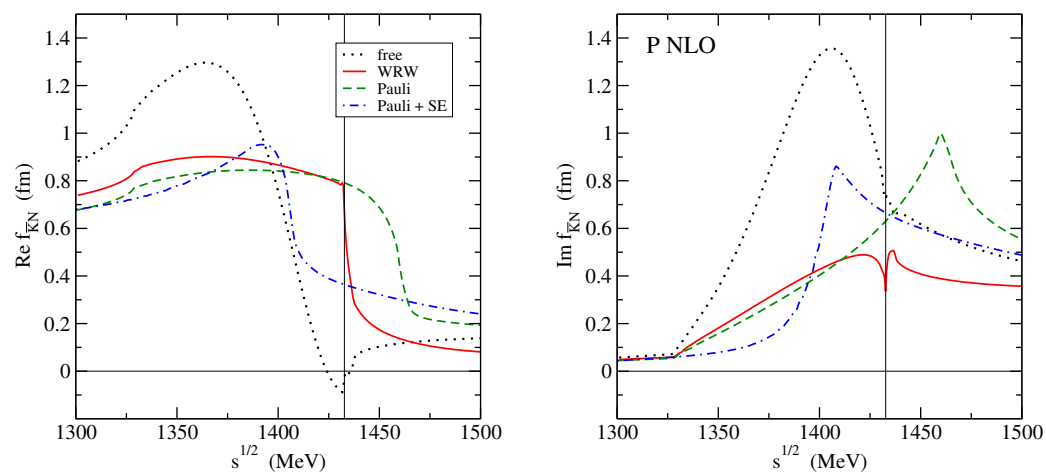

Figure 2. Energy dependence of free-space (dotted line) $f_{\bar{K} N}=\frac{1}{2}\left(F_{K^{-} p}+F_{K^{-} n}\right)$ amplitude compared with WRW modified amplitude (solid line), Pauli (dashed line), and Pauli + SE (dot-dashed line) modified amplitude for $\rho_{0}=0.17 \mathrm{fm}^{-3}$ in the P NLO model.

\section{Results}

With the formalism introduced above we performed self-consistent calculations of $K^{-}$quasi-bound states in nuclei across the periodic table. In Figure 3, we present the $1 s K^{-}$binding energies $B_{K^{-}}$and corresponding widths $\Gamma_{K^{-}}$as a function of the mass number calculated for different baryon-meson interaction models. The $\mathrm{K}^{-}$binding energies exhibit considerable model dependence, nevertheless their A dependence is very similar in all models considered. The $K^{-}$widths show rather weak A dependence except for the M2 model. The KM NLO model predicts widths twice as large as the P NLO and M1 models. Within the Bonn models B2 and B4, we did not succeed to obtain any bound states since the real parts of the $K^{-} N$ amplitudes are weakly attractive or even repulsive below threshold (see Figure 1). In Figure 4, the $K^{-}$spectrum in ${ }^{40} \mathrm{Ca}$ calculated for various models is shown. Again, the $K^{-}$binding energies strongly depend on the model used. In the P NLO and KM NLO
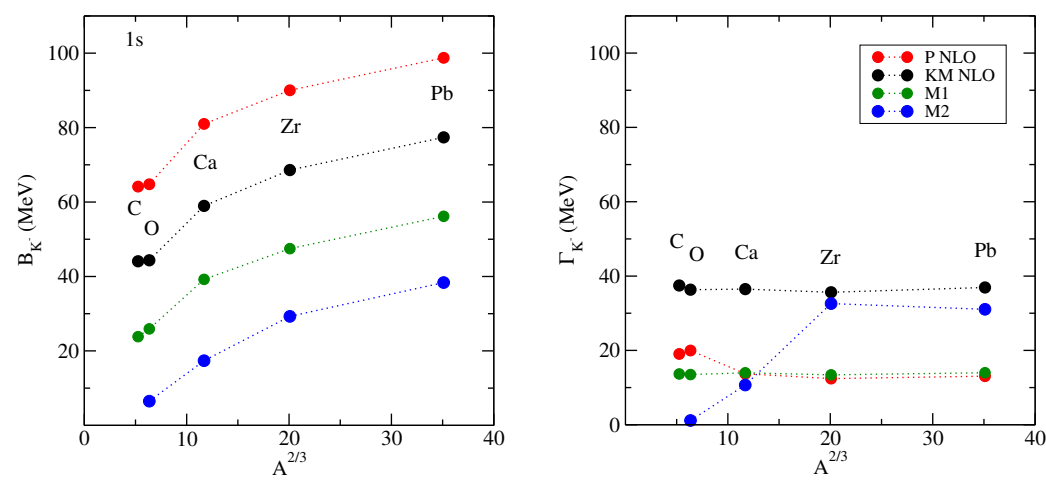

Figure 3. 1s $K^{-}$binding energies (left) and corresponding widths (right) in various nuclei calculated selfconsistently in the P NLO, KM NLO, M1, and M2 models. $K^{-} N N \rightarrow Y N(Y=\Lambda, \Sigma)$ decay modes are not considered. 

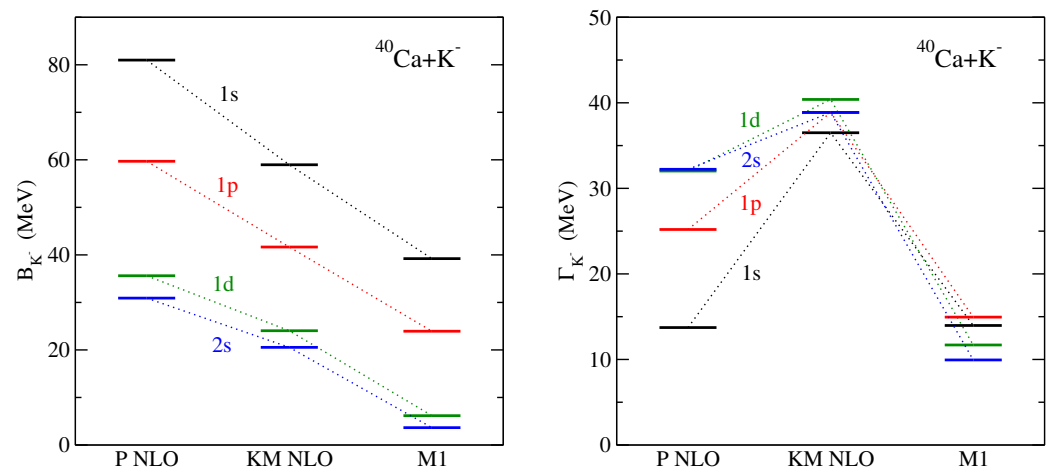

Figure 4. $K^{-}$binding energies (left) and widths (right) in $s, p$ and $d$ levels in ${ }^{40} \mathrm{Ca}$ calculated self-consistently in the P NLO, KM NLO, and M1 models. $K^{-} N N \rightarrow Y N$ decay modes are not considered.

models, the $K^{-}$in the $1 s$ state has the smallest $K^{-} N \rightarrow \pi Y$ conversion width due to the considerable energy shift towards the $\pi \Sigma$ threshold. The $K^{-}$widths of excited states grow as the $\sqrt{s}$ moves farther from the $\pi \Sigma$ threshold. However, in the M1 model the widths follow the opposite trend. It is due to the fact that $\sqrt{s}$ is much closer to the $\bar{K} N$ threshold where the imaginary part of the $K^{-} p$ amplitude starts to decrease (see Figure 1).

It is to be noted that the core polarization caused by $\mathrm{K}^{-}$was not taken into account in the present calculations. Previous studies of kaonic nuclei [8] found this effect quite mild, adding up to $5 \mathrm{MeV}$ to $K^{-}$binding energies. Moreover, the annihilation of $K^{-}$on 2 nucleons [8] should be considered as well, since it increases the width by almost $50 \mathrm{MeV}$ and, consequently, even the $1 s$ state $K^{-}$widths become comparable with the binding energies. We intend to include both effects mentioned above in the upcoming calculations.

We wish to thank A. Gal and E. Friedman for fruitful discussion, and M. May for providing us with the free $\bar{K} N$ scattering amplitudes. This work was supported by the GACR Grant No. P203/15/04301S. J. Hrtánková acknowledges financial support from CTU-SGS Grant No. SGS16/243/OHK4/3T/14.

\section{References}

[1] A. Cieply, J. Smejkal, Nucl. Phys. A 881, 115 (2012)

[2] Y. Ikeda, T. Hyodo and W. Weise, Nucl. Phys. A 881, 98 (2012)

[3] Z. H. Guo, J. A. Oller, Phys. Rev. C 87, 035202 (2013)

[4] M. Mai and U.-G. Meißner, Nucl. Phys. A 900, 51 (2013)

[5] M. Bazzi et al (SIDDHARTA Collaboration), Phys. Lett. B 704, 113 (2011)

[6] T. Wass, M. Rho and W. Weise, Nucl. Phys. A 617, 449 (1997)

[7] A. Cieplý, E. Friedman, A. Gal, D. Gazda and J. Mareš, Phys. Lett. B 702, 402 (2011)

[8] D. Gazda, J. Mareš, Nucl. Phys. A 881, 159 (2012) 